\title{
La consolidation atmosphérique
}

\author{
Vacuum consolidation
}

\author{
J.-M. COGNON
}

Ingénieur ECP. Ménard soltraitement*

Rev. Franç. Géotech. n 57, pp. $37-47$ (octobre 1991)

\section{Résumé}

La consolidation atmosphérique est une variante du préchargement d'un sol argileux par un remblai classique associé à un réseau de drains verticaux. L'article présente le principe de la méthode de calcul de consolidation atmosphérique et montre les différences importantes entre les deux méthodes.

Principalement, la consolidation atmosphérique étant de type isotrope, l'amélioration des caractéristiques de sol est différente et la rupture et le fluage latéral sont impossibles.

En fin d'article les résultats globaux d'un certain nombre de réalisation sont présentés.

\footnotetext{
Abstract

Vacuum consolidation is an alternative to surcharging of cohesive soil by means of a conventional fill associated to vertical drains.

This article introduces to calculation methods of vacuum consolidation and compares the basic differences.

Basically, vacuum consolidation beeing isotropic, the variations of soil caracteristics follow different laws, failure as well as plastic flow will not occur.

The article is highlighted by a series of recent case histories.
} 


\section{GÉNÉRALITÉS}

Le principe de cette méthode apparait simple au premier abord comme on peut le constater par le calcul ci-dessous :

Supposons un sol déjà consolidé sous une faible hauteur de remblai au-dessus duquel est mise en œuvre une membrane étanche refermée sur des tranchées étanches descendue au moins au niveau de la nappe (fig. 1)

Il suffit pour comprendre le mécanisme de prendre en compte la pression atmosphérique dans les contraintes vraiment totales cette fois et dans les pressions interstitielles.

Avant de réaliser le vide à une profondeur $z$ :

$$
\begin{aligned}
& \sigma_{\mathrm{T}}=\gamma z+\gamma_{\mathrm{r}} \cdot \mathrm{h}+\mathrm{P}_{\mathrm{a}}=\sigma_{\mathrm{t}}+\mathrm{P}_{\mathrm{a}} \\
& \mathrm{u}_{\mathrm{T}}=\mathrm{z} \gamma_{\mathrm{w}}+\mathrm{P}_{\mathrm{a}}=\mathrm{u}_{\mathrm{t}}+\mathrm{P}_{\mathrm{a}} \\
& \sigma_{\mathrm{i}}=\sigma_{\mathrm{T}}-\mathrm{u}_{\mathrm{T}}=\sigma_{\mathrm{t}}-\mathrm{u}_{\mathrm{t}}=\mathrm{z} \gamma^{\prime}+\mathrm{h} \gamma_{\mathrm{r}}
\end{aligned}
$$

Les indices T étant affectés aux contraintes y compris la pression atmosphérique et $\mathrm{t}$ aux contraintes classiques, $\sigma_{i}^{\prime}$ étant la contrainte effective initiale avant de faire le vide.

Si le vide est maintenu sous la membrane pendant un temps infini $\sigma_{\mathrm{T}}$ ne change pas, tandis que $\mathrm{u}_{\mathrm{T}}$ devient $u_{t}=2 \gamma_{w+0}$ :

d'où $\sigma_{f}$ en fin de consolidation devient :

$$
\sigma_{f}^{\prime}=\sigma_{\mathrm{T}}-\mathrm{u}_{\mathrm{t}}=\sigma_{\mathrm{t}}+\mathrm{Pa}
$$

la contrainte effective est donc augmentée de la valeur de la pression atmosphérique si le rendement des pompes est de 1 . Ce raisonnement est valable pour $\sigma_{h}^{\prime}$, on est donc en présence d'une augmentation des contraintes isotrope.

\section{CONDITIONS LIMITES}

Le raisonnement précédent n'est valable qu'aux quatre conditions suivantes:

1. la valeur $\mathrm{z}$ doit être faible par rapport à la largeur ; 2. il faut $r<<r_{0}$ pour ne pas avoir d'effet de bord ;
3. il faut $\mathrm{h}$ faible sinon le raisonnement devient faux car la nappe va monter dans le remblai drainant et provoquera une diminution de $\sigma_{f}^{\prime}$ par déjaugeage du remblai ;

4. le vide doit être réalisable avec des moyens économiques, c'est-à-dire qu'il faut peu de venue d'eau, ce procédé est donc intéressant dans les sols argileux, mais ce sont les seuls sur lesquels on envisage des méthodes d'amélioration des caractéristiques par préchargement.

En effet, s'il n'est pas pris de précaution particulière, le schéma d'écoulement en cas d'une nappe à une certaine profondeur sera celui indiqué sur la figure 3 partie droite.

Loin des bords dans la zone sans drain, en supposant que la nappe du sable inférieure est à la même charge hydraulique que l'argile et que le rendement de la pompe à vide, y compris les pertes de charge dans les sables, soit de 0,8 , on peut calculer l'augmentation de contrainte effective.

En prenant l'origine au point $\mathrm{O}$, la charge hydraulique en A reste identique puisque la membrane n'a pas une dimension infinie et vaut:

$$
\begin{aligned}
& \mathrm{h}=\frac{\mathrm{u}}{\gamma_{\mathrm{w}}}+\mathrm{z}=12+0=12 \\
& \text { et } \mathrm{h}_{\mathrm{T}}=12+10=22
\end{aligned}
$$

en tenant compte de la pression atmosphérique...

Nous avons, avant pompage, la même valeur en B par hypothèse :

$$
h_{B}=(10+12=22) \text {. }
$$

Après pompage $h_{A}$ reste la même, $h_{B}$ devient avec les valeurs prises à la gauche de la figure:

$$
\frac{\mathrm{u}}{\gamma_{\mathrm{w}}}+\mathrm{n} \cdot \mathrm{P}_{\mathrm{a}}+\mathrm{z}=3+0,2 \times 10+12=17
$$

l'augmentation de contrainte effective en $\mathrm{B}$ sera donc limitée à $50 \mathrm{KPa}$; et $80 \mathrm{KPa}$, si l'on ne laisse pas monter l'eau dans le remblai.

Cette augmentation de contrainte sera nulle en $A$ et la variation entre $A$ et $B$ sera linéaire en fin de consolidation quand l'écoulemement sera devenu permanent.

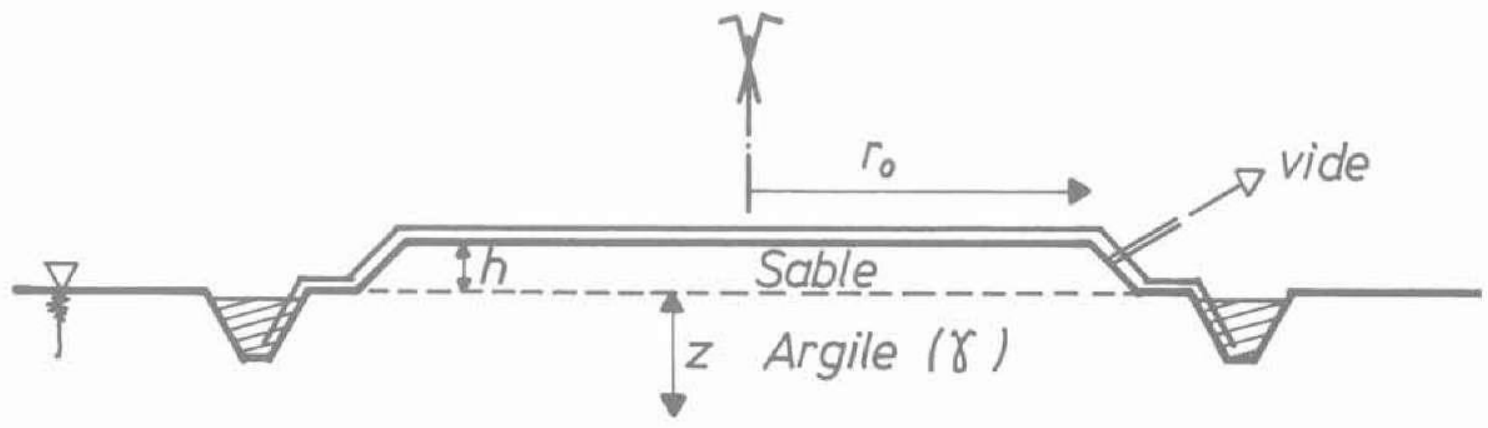

Fig. 1. - Principe de la consolidation atmosphérique.

Fig. 1. - Vaccum consolidation principle. 


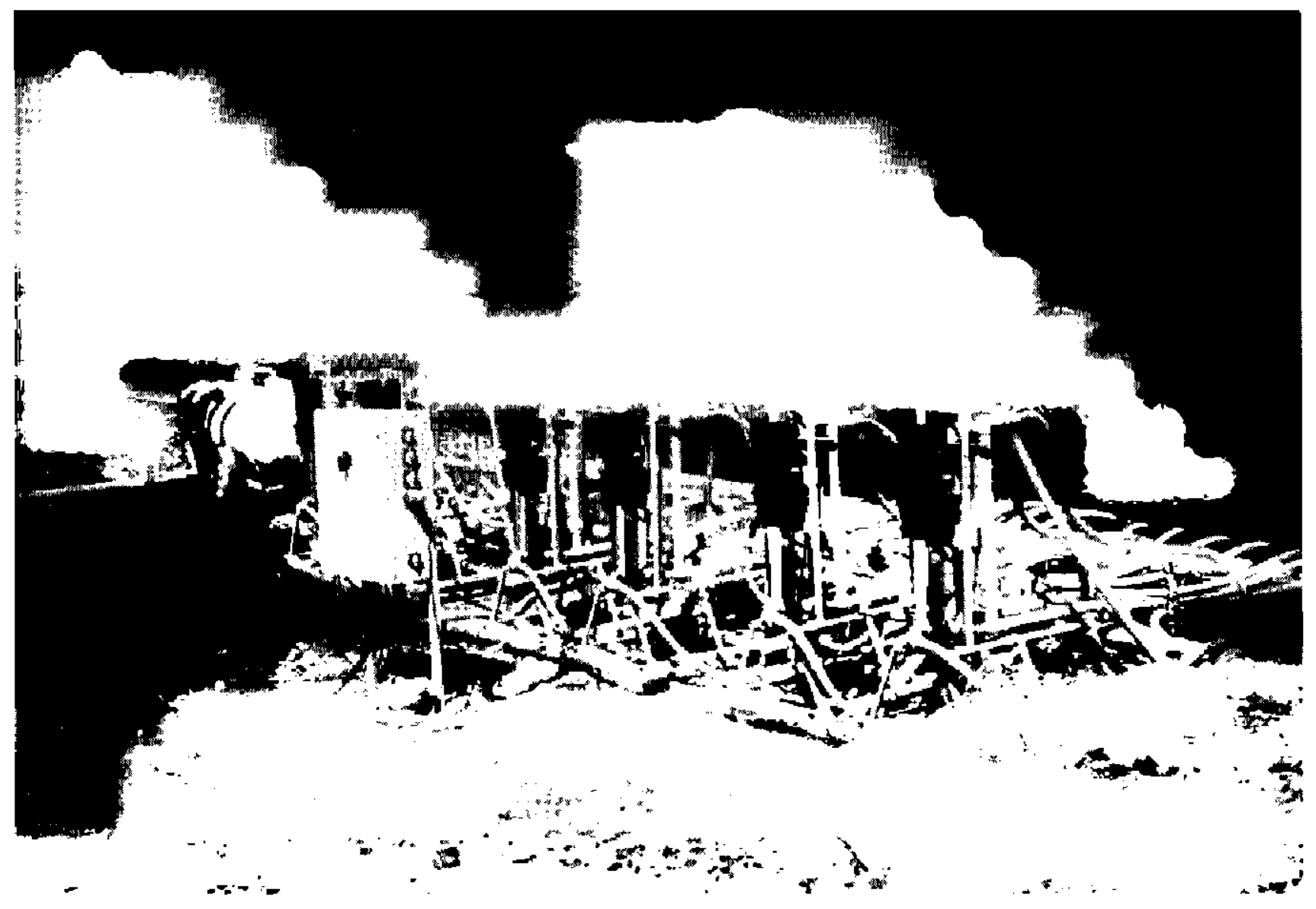

Fig. 2. - Vue générale d'un chantier.

Fig. 2. - General view of a site.

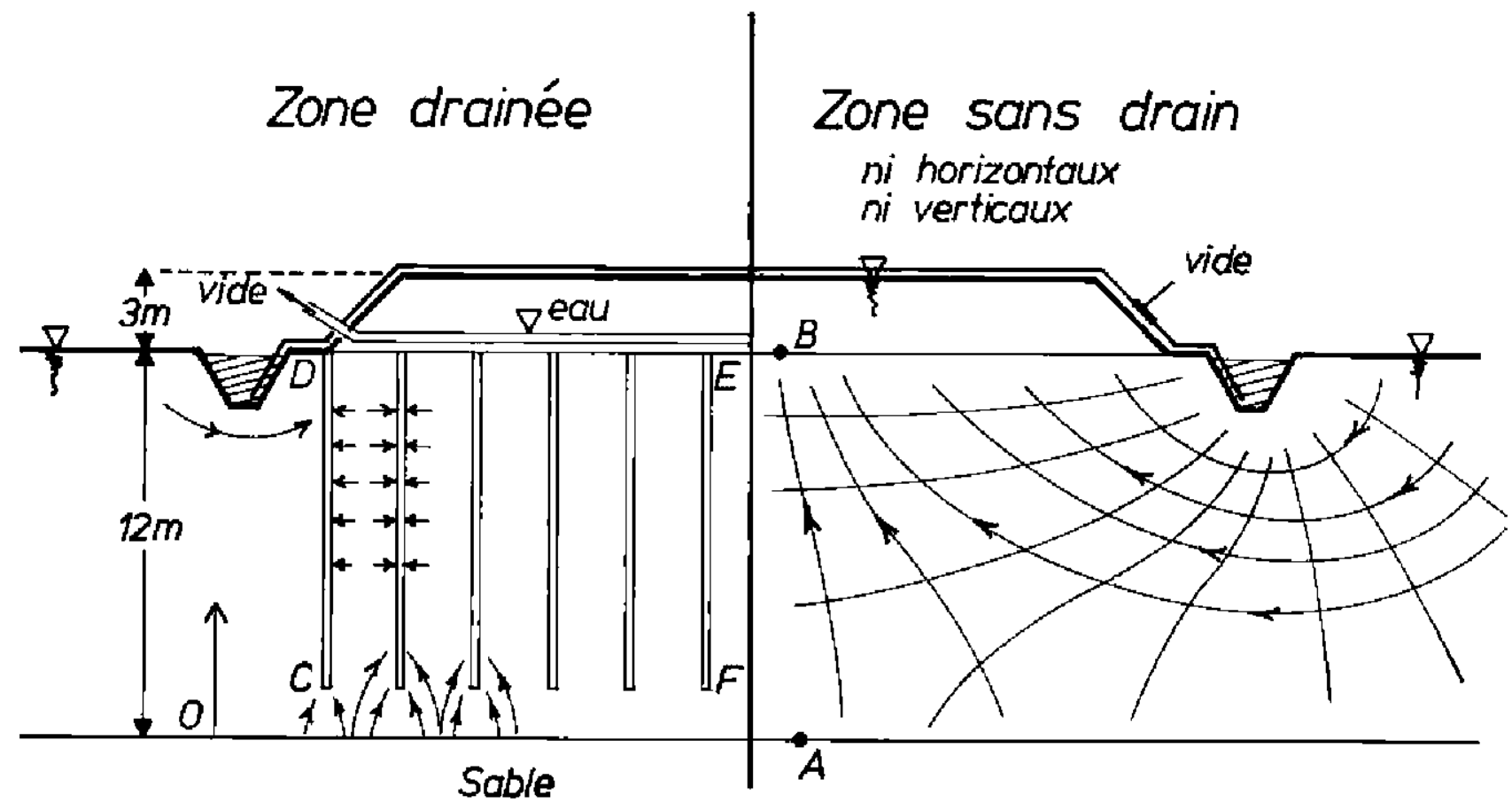

Fig. 3. - Ecoulement de l'eau avec et sans drain.

Fig. 3. - Flow tines with and without drains, 
Dans la partie gauche de la figure, si l'on empeche la nappe de monter dans les remblais et si l'on dispose des drains verticaux. l'écoulement va être modifié. En effet, le gradient le plus fort sera toujours horizontal saut dans la zone située entre la couche de sable inférieure et la base des drains.

On se retrouve dans cette configuration dans le cas de calcul initial et laugmentation de contrainte effec. tive será égale au rendement près à la pression atmosphérique, soit $80 \mathrm{KPa}$ en tout point du massif CDEF.

Les effets de bord dans la zone du point $C$ sont réduits au minimum en diminuant la maille des drains en périphérie, comme on le verra plus loin.

Techniquement, il est difficile d'empêcher la nappe de monter dans les remblais, c'est d'ailleurs la première cause d'échec avec la réalisation de drains verticaux descendant dans une couche perméable, des tentatives réalisées dans le passé ; les collecteurs se sont alors pratiquement remplis d'eau et le vide ne put se réaliser correctement. C'est pour éviter ces déboires que le système breveté prévoit la réalisation concommittante d'un rabattement sous la membrane, celui-ci peut étre réalisé de deux façons:

1. réalisation de drains horizontaux profonds ou tranchées drainantes (5 $\mathrm{m}$ de protondeur) :

2 . système de vidange des drains avec pompes plastiques incorporées dans chaque drain pour mointenir l'eau à $5 \mathrm{~m}$ de profondeur environ.

Au niveau théorique, l'approche est la même et peut se présenter comme suit.

\section{ASPECT THÉORIQUE}

\subsection{Diagramme de consolidation classique}

Pour comprendre l'intérêt de superposer le rabattement avec laction du vide, it est utile de présenter la consolidation en portant sur un axe vertical, les pressions interstitielles et les contraintes comme expliqué sur la tigure 5 dans le cas d'une consolidation classique dans lhypothèse unidimensionnelle de TERZAGHI, les nappes supérieures et inférjeures étant à la même charge hydraulique.

Avant toute mise en place de surcharge, à partir d'un point $O$ de la surface libre de la nappe. on trace une droite a $45^{\circ}$, de cette façon en l'absence de consolidation en cours la pression interstitielle a une profondeur z en-dessous de la nappe, est donnée par la bongueur $\mathrm{MN} \cdot \gamma \mathrm{w}+\mathrm{Pa}_{\mathrm{a}}$.

Si l'on veut réaliser un préchargement avec un remblaj de grande dimension (cas unidimensionnel) au temps $\mathrm{t}=0$ la contrainte $\Delta \sigma$ est transmise à l'eau et la pression interstitielle augmente donc de $\Delta \sigma$. La consolidation s'opérant petit à petit. la surpression de chaque point de l'argile sera donnée par la courbe $A N ' B$ en l'absence de drain pour revenir à $A B$ pour un temps théoriquement infini (figure 6).

La surface AA'B'N'A représente le diagramme de consolidation et la longueur N'P l'augmentation de la contrainte effective pour le point $\mathrm{M}$ situé à une profondeur $z$ en cours de consolidation. In fine $N$ ' rejoint $N$ et l'augmentation de contrainte effective est la même en tout point de l'argile et vaut N P c'est-àdire $\Delta \sigma$,
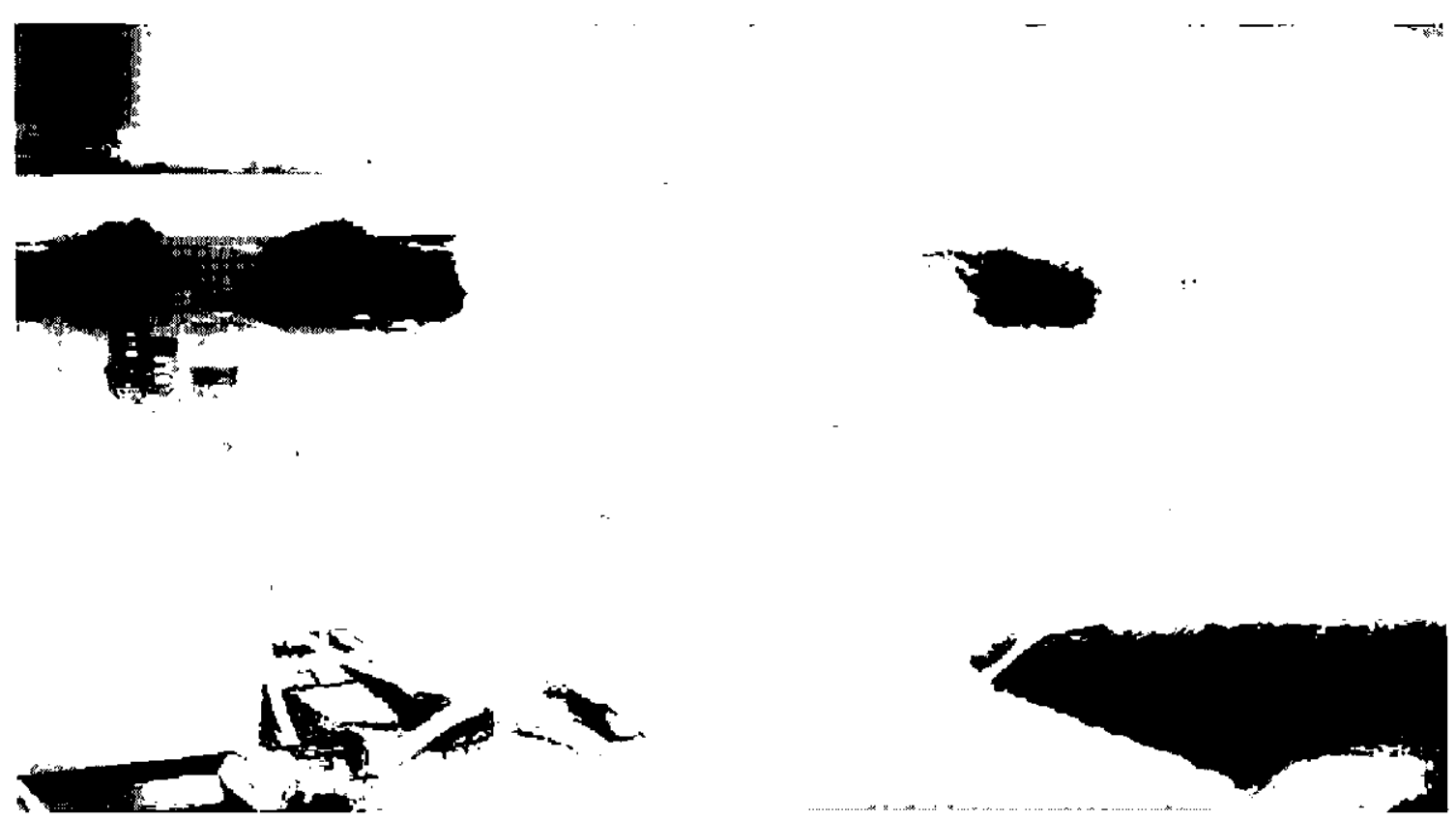

Fig. 4. - Systeme de conjonction entre les drains de surface, les drains profonds et les pompes à vide.

Fig. 4. - Connections between surface horizontal drains, vertical drains and vaccum pumps. 


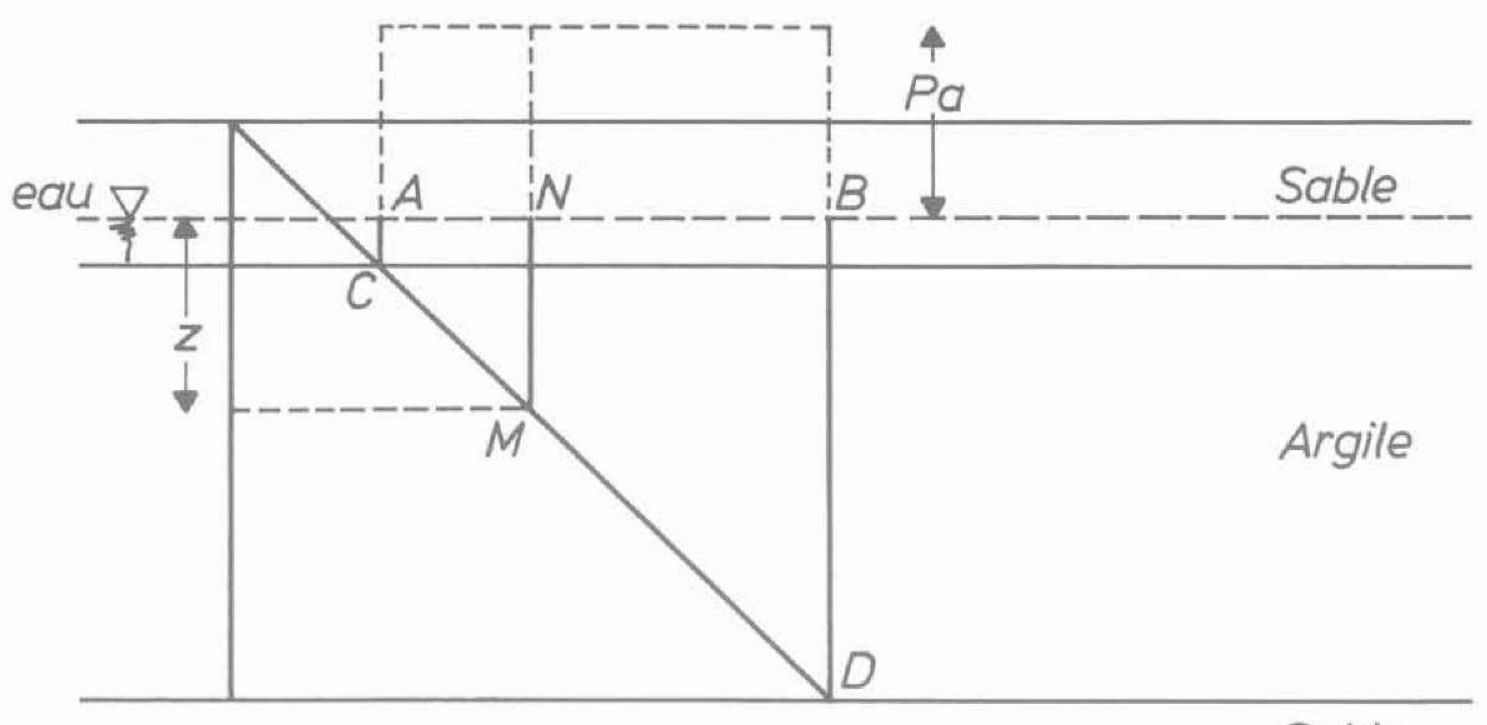

Sable

Fig. 5. - Visualisation de la pression interstitielle. Fig. 5. - Representation of pore pressure.

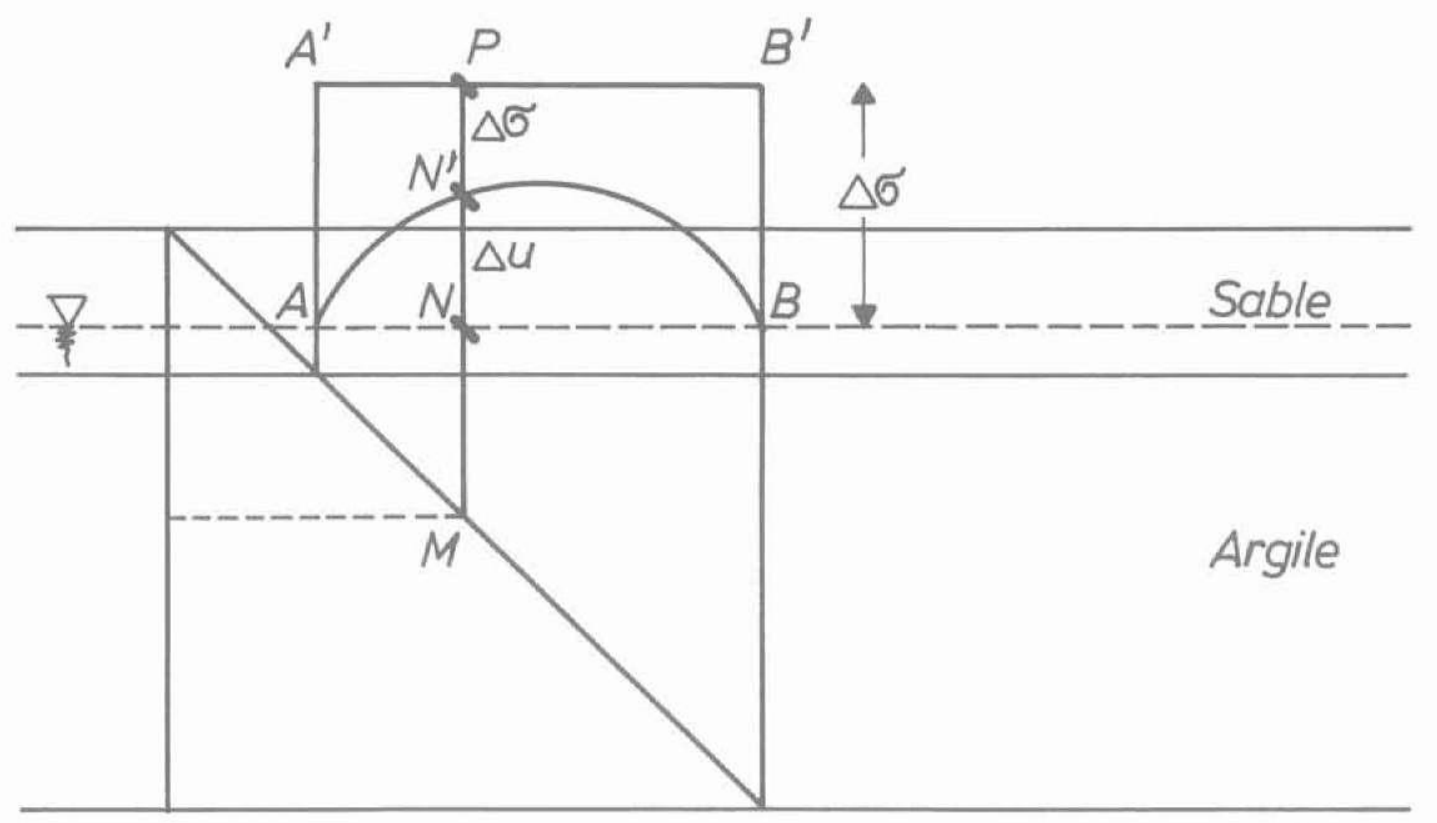

\section{Sable}

Fig. 6. - Evolution de la pression interstitielle dans la théorie unidimensionnelle de TERZAGHI. Fig. 6. - Pore pressure variation according to TERZAGHI one dimensional consolidation theory.

Dans le cas d'une surcharge de dimensions finies (par exemple un bac de pétrole apportant une contrainte de 80 de diamètre égal à l'épaisseur de l'argile) le schéma est celui de la figure 7 avec l'hypothèse simplicatrice d'un comportement élastique du squelette solide et d'une consolidation unidimensionnelle, même si dans ce cas elle est peu réaliste, NP représente toujours l'augmentation de contrainte effective à la profondeur $z$. A'B' représente la courbe classique de BOUSSINESQ.

\subsection{Diagramme de consolidation par le vide}

En restant dans le même principe de graphique, on peut maintenant mieux comprendre trois cas déjà étudiés.

a. Consolidation atmosphérique sans drain avec nappe à une distance finie (fig. 8).

Dans ce cas, il existe une loi linéaire de l'augmentation de la contrainte effective. Ce schéma n'étant vala- 


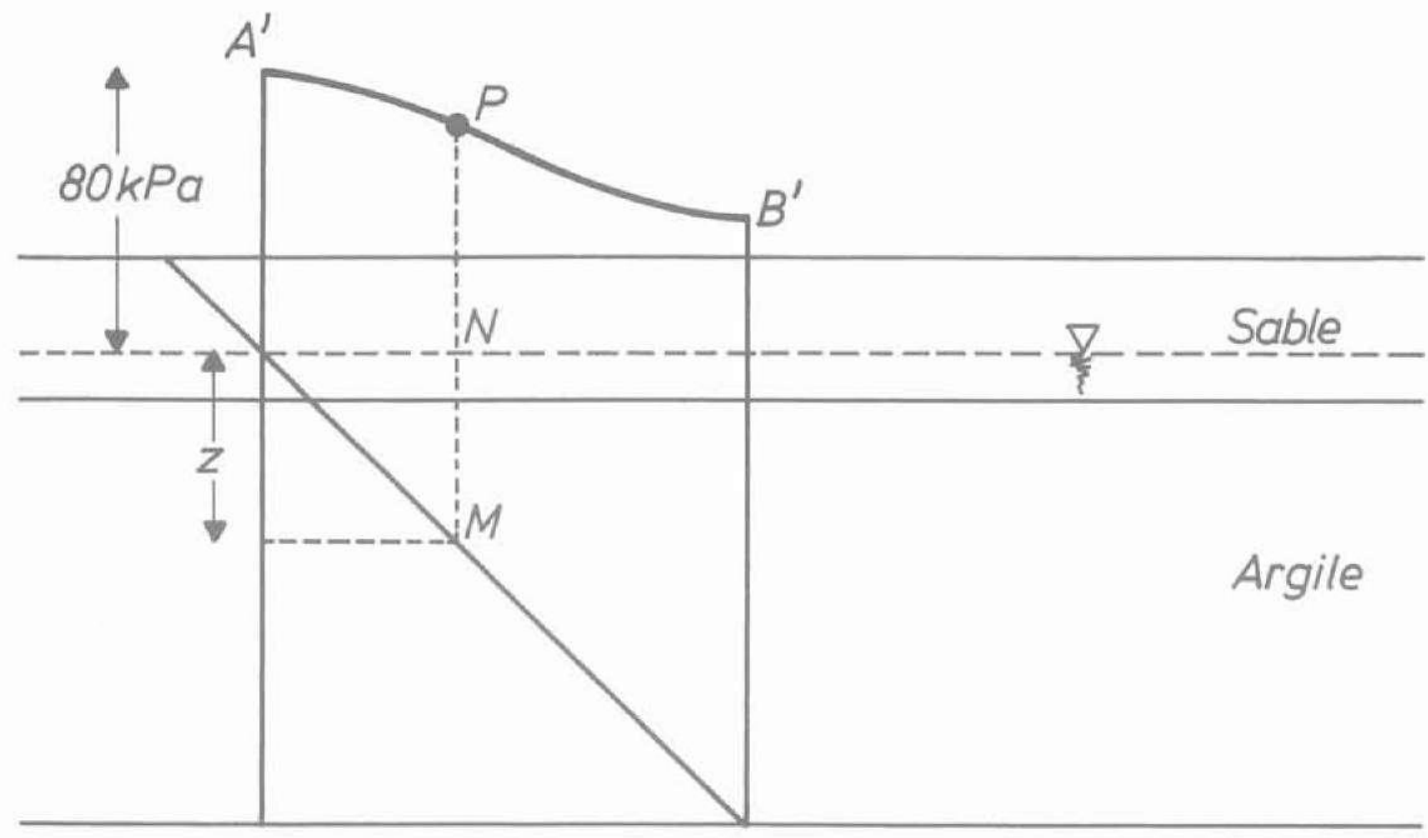

Sable

Fig. 7. - Schéma unidimensionnel avec contrainte diminuant avec la profondeur. Fig. 7. - One dimensional graph of stress decreasing with depth.

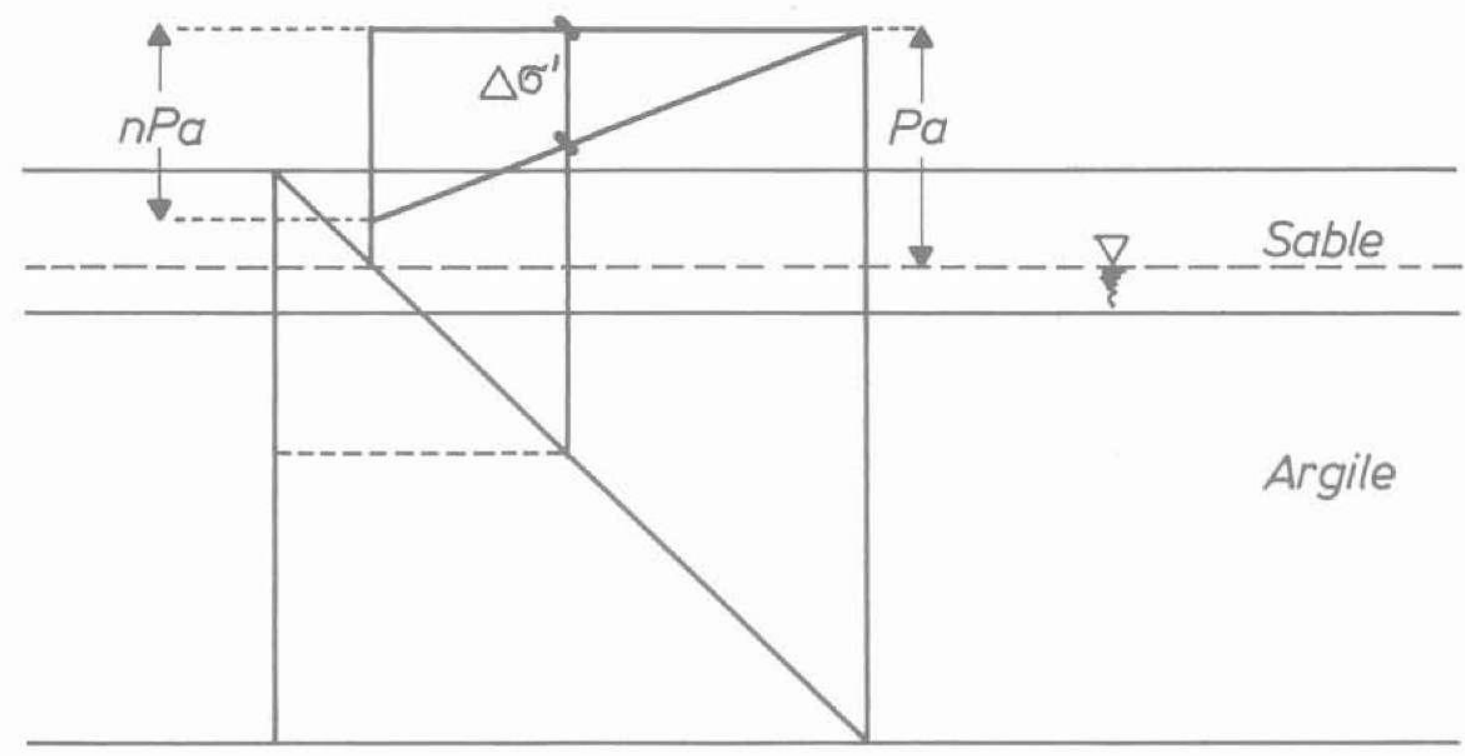

Sable

Fig. 8. - Schéma unidimensionnel du pompage sans drains.

Fig. 8. - One dimensional graph of vacuum application without drains.

ble que dans la zone centrale en ne tenant pas compte des effets de bord étudiés plus loin.

b. Consolidation atmosphérique avec drain arrêté avant une couche drainante. Ce cas est un peu théorique comme on l'a vu précédemment pour des problèmes purement technologiques (fig. 9).

Par contre, même si la dimension de la membrane est finie, il n'y a pas comme dans le cas d'une sur- charge classique, même avec des drains de diminution de la contrainte effective créée en fonction de la profondeur comme indiqué dans la figure 7.

c. Consolidation atmosphérique combinée avec un " rabattement ». Dans le cas d'un « rabattement " de $5 \mathrm{~m}$, on se trouve en présence d'une augmentation de l'efficacité de 0 à $5 \mathrm{~m}$ dans les 5 premiers mètres. Ce supplément de gain du gradient d'écoulement et 


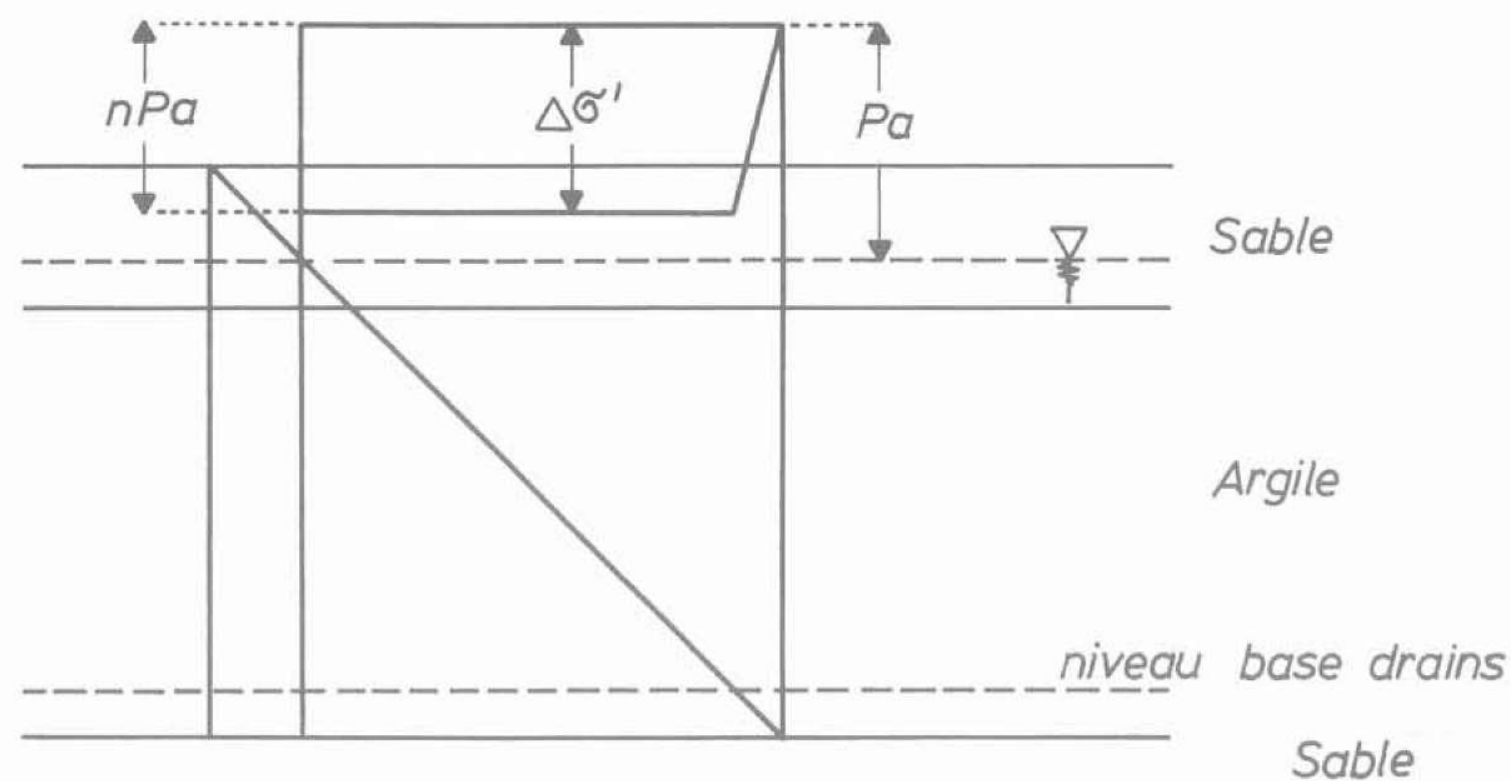

Fig. 9. - Schéma unidimensionnel du pompage avec drain.

Fig. 9. - One dimensional vacuum application with drains.

de la contrainte effective finale restant constant ensuite jusqu'à la base des drains.

\section{DÉFORMATION}

L'étude des déformations est intéressante principalement pour approcher la connaissance des effets de bords qui sont évidemment très différents de ceux d'un préchargement classique puisque l'eau circule dans lautre sens, ce qui provoque une inversion des déformations relatives horizontales.

Nous n'étudierons que le cas à deux dimensions (bande sous vide) car elle est la seule qui possède une solution analytique en considérant une loi de comportement élastique. Le résultat, a priori intuitif, est que la contrainte effective dans un milieu non pesant élastique par une dépression créée en surface est de type isotrope $\sigma_{1}=\sigma_{2}$.
On constate d'autre part que la valeur de cette contrainte est égale à la moyenne des deux contraintes principales calculées par BOUSSINESQ dans le cas d'une bande souple chargée uniformément avec la même valeur que la dépression créée (fig. 10).

$$
\begin{gathered}
\sigma_{1}, \sigma_{2}=\frac{\mathrm{P}}{\pi}(\theta \pm \sin \theta) \sigma \text { isotrope }=\frac{\mathrm{P}}{\pi} \theta \\
\frac{\sigma_{1}, \sigma_{2}}{2}=\frac{\mathrm{P}}{\pi} \theta
\end{gathered}
$$

les déformations engendrées par ces contraintes en fin de consolidation sont données par la relation :

$\mathrm{W}(\mathrm{x}, \mathrm{H})=[1-\nu(1+2 \nu)] \frac{\mathrm{n} \cdot \mathrm{P}_{\mathrm{a}}}{\pi \mathrm{E}} \cdot \mathrm{F}(\mathrm{x}, \mathrm{H})$ avec ;

$$
F(x, H)=H \operatorname{Arctg} \frac{b-x}{H}+H \operatorname{Arctg} \frac{b+x}{H}
$$

\section{SURCHARGE}

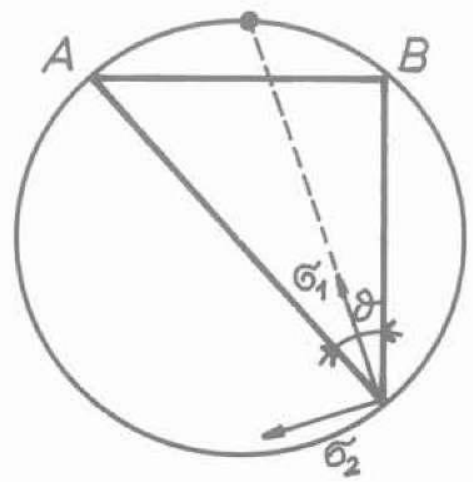

\section{DEPRESSION}

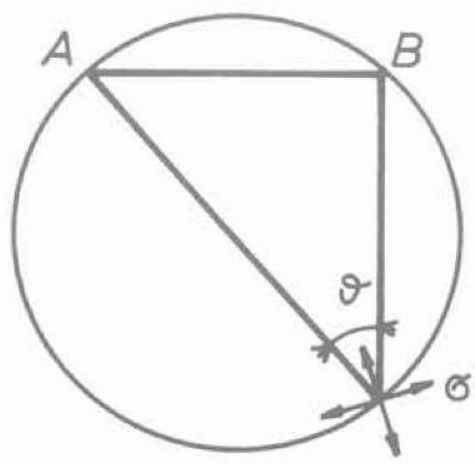

Fig. 10. - Etat de contraintes à deux dimensions. Fig. 10. - Two dimensional state of stress. 


$$
\begin{aligned}
& +\frac{b-x}{2} \cdot L\left[1+\frac{H^{2}}{(b-x)^{2}}\right] \\
& +\frac{b+x}{2} L\left[\frac{1+H^{2}}{(b+x)^{2}}\right]
\end{aligned}
$$

avec les dêfinitions données dans la figure 11 .

l] faut noter que, comme pour un remblai le tassement tend vers l'infini si l'épaisseur tend vers l'infini.

Cette méthode de calcul permet de résoudre le problème du tassement à proximité de la membrane. En effet, sous la membrane le sol se consolide sous l'action d'une contrainte isotrope. les grains du sol à la périphérie ont donc tendance à se rapprocher du centre alors que c'est le contraire dans le cas d'une surcharge classique. La figure 12 montre l'allure du tassement dans les trois cas:

- surcharge par temblai :

- consolidation atmosphérique sans drain :

- consolidation atmosphérique avec dratss.

Cette dernière méthode peut donc être appliquée à proximité de pieux sans risque de rupture.

De plus, dans le cas de sol très compressible, le préchargement par un remblai présente l'inconvénient d'apporter une contrainte qui diminue en fonction du tassement ; la base du remblai en passant sous la nappe se trouve déjaugée et la contrainte apportée diminue.

Dans le cas de pompage sous vide avec drains horizontaux, le remblai situé sous la membrane reste évidemment sec.

\section{5. ÉTUDE DE LA CONSOLIDATION RÉELLE}

\subsection{Dans les argiles}

Pour approcher de plus près la réalité des phénomènes, il taut quitter le domaine élastique et effectuer une comparaison entre un préchargement classique et un préchargement par la consolidation atmosphérique.

Rappel 5.1. dans les coordonnées:

$$
p^{\prime}=\frac{\sigma_{1}^{\prime}+\sigma_{3}^{\prime}}{2} q^{\prime}=\frac{\sigma_{1}^{\prime}-\sigma_{3}^{\prime}}{2}
$$

Lors d'un essat triaxial (CIU) le schéma des contraintes effectives suit une courbe $A B$ et si ensuite on laisse la consolidaiton opérer, le point $\mathrm{B}$ suit le chemin $\mathrm{AC}$,

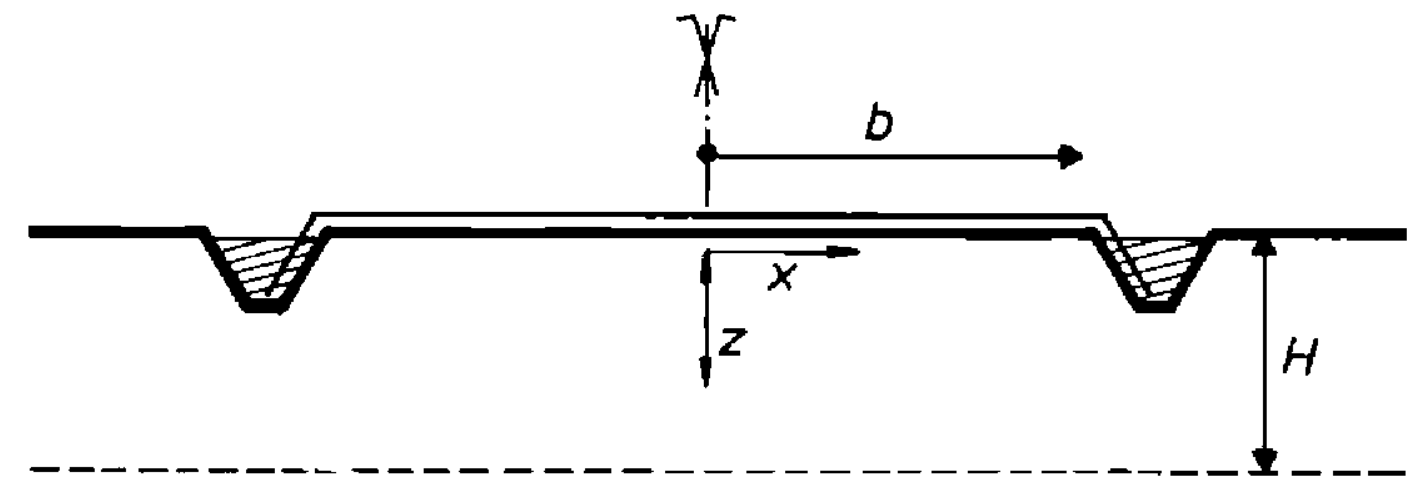

Fig. 11. - Notations à deux dimensions.

Fig. 11. - Definition of two dimansional parameters.

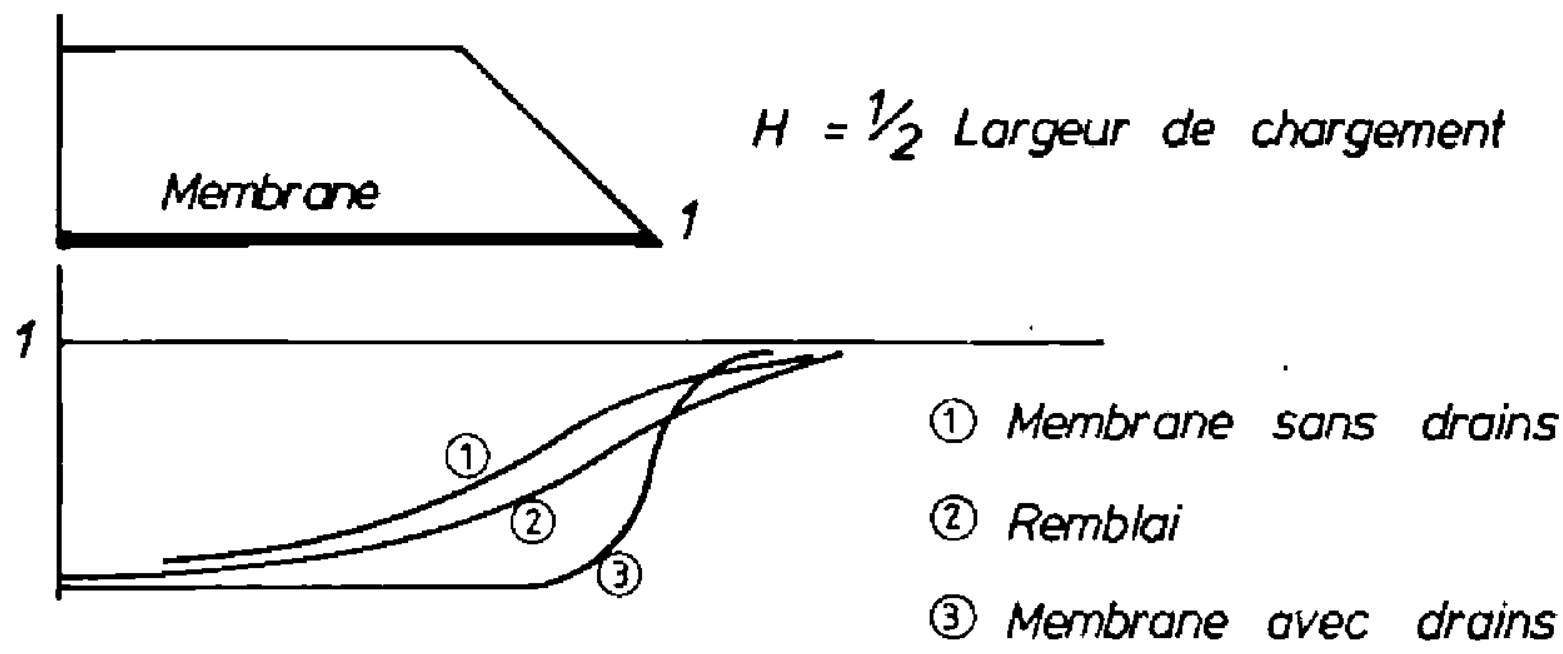

Fig. 12. - Forme de la base du remblai dans 3 cas de chargement. Fig. 12. - Basis shape of fill as function of three loading cases. 


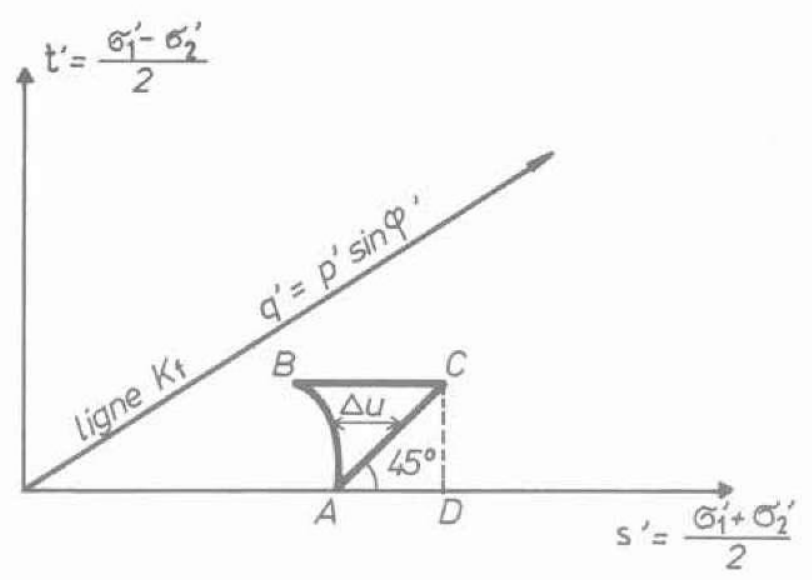

Fig. 13. - Principe du diagramme p, q. Fig. 32. - P, q diagram.

$$
\text { en appelant } A=\frac{\Delta u}{\Delta \sigma_{1}}=\frac{B C}{2 C D}
$$

on peut écrire $\Delta \mathrm{u}=\Delta \sigma_{3}+\mathrm{A}\left(\Delta \sigma_{1}-\Delta \sigma_{3}\right)$.

En première approximation on peut considérer que le chemin des contraintes est du même type sous un remblai au moins près du centre.

5.2. Dans la méthode cedométrique on s'interdit les déplacements horizontaux, donc:

$$
\frac{\mathrm{q}^{\prime}}{\mathrm{p}^{\prime}}=\mathrm{cte}
$$

et le chemin des contraintes suit la "ligne Ko " de pente :

$$
\frac{1-\mathrm{K}_{\mathrm{o}}}{1+\mathrm{K}_{\mathrm{o}}}, \mathrm{K}_{\mathrm{o}} \text { étant le rapport } \frac{\sigma_{3}^{\prime}}{\sigma_{1}^{\prime}}
$$

cette ligne $\mathrm{K}_{\mathrm{o}}$ coupe le plan Op' ligne $\mathrm{Kf}$ en deux zones.

Au-dessus les déplacements horizontaux sont vers l'extérieur e $\mathrm{h}<0$ sur la ligne $e \mathrm{~h}=0$ par définition et en dessous $e \mathrm{~h}>0$.

Le chemin de contrainte réelle sera donc $A B C$ alors que celui pris en compte par la méthode œedométrique sera $\mathrm{AD}, \mathrm{C}$ et $\mathrm{D}$ ayant le même $\sigma_{1}^{\prime}$.

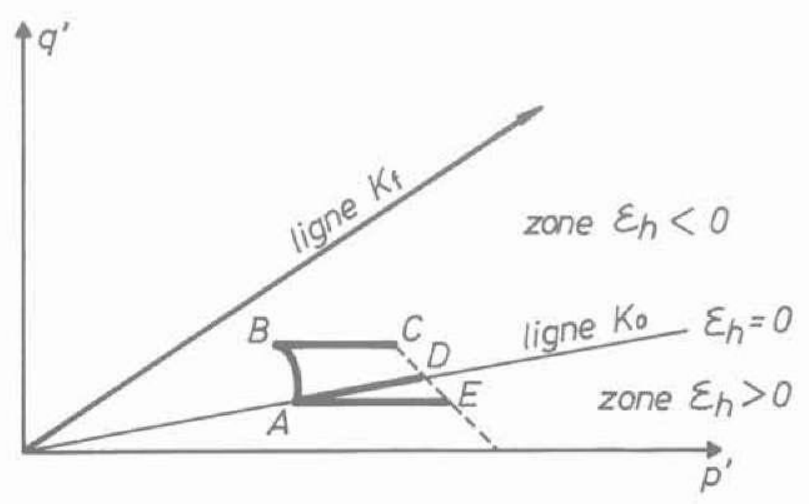

Fig. 14. - Schéma des deux systèmes de consolidation. Fig. 14. - Stress path of two processes of consolidation.
Dans le cas de la consolidation atmosphérique pour un essai triaxial aucun déplacement latéral n'étant empêché, on se retrouve évidemment avec $\Delta \sigma_{v}^{\prime}=\Delta \sigma_{\mathrm{h}}^{\prime}$. c'est-à-dire sans déviateur et le chemin de contrainte est horizontal de type $\mathrm{AE}$.

Les points $\mathrm{CDE}$ étant sur une droite à $45^{\circ}$ correspondant à la même contrainte $\sigma_{\mathrm{v}}^{\prime}$.

Sur un chantier réel à proximité du remblai de surface il y a un effet de frettage entre la couche supérieure et l'argile, ce qui empêchera le déplacement horizontal et le chemin de contrainte sera AD. Globalement les chemins de contraintes se situeront dans l'angle $\mathrm{AD}, \mathrm{AE}$ c'est-à-dire dans la zone correspondant à un $\epsilon_{h}$ positif alors que dans le cas d'un remblai il $y$ a toujours $\epsilon_{\mathrm{h}}$ négatif c'est-à-dire avec un déplacement latéral vers l'extérieur, la séparation entre les systèmes étant la droite $A D$ correspondant à l'œdomètre c'est-à-dire $\epsilon_{\mathrm{h}}$ égal à zéro.

On peut donc mettre une membrane à côté de bâtiments sur pieux sans risque de rupture comme on peut le voir sur la photo page suivante.

\subsection{Augmentation de résistance au cisaillement dans les sols fins}

Comme on l'a vu dans le calcul des contraintes effectives, on se trouve proche d'un essai CIU donc on pourra compter sur une augmentation de $\mathrm{Cu}$ déduit de ces essais alors que dans un préchargement classique on devrait prendre des résultats en réalisant des essais CAU ou la contrainte moyenne et donc la variation d'indices des vides finale est plus faible.

\subsection{Augmentation de la résistance au cisaillement dans les sols granulaires}

Comme on l'a vu précédemment, ce procédé ne peut évidemment pas provoquer de rupture, bien au contraire, puisque le coefficient de sécurité augmente au fur et à mesure de la consolidation.

Si l'on veut réaliser un préchargement au-dessus d'un remblai pour provoquer une accélération du tassement, il sera préférable de mettre le remblai le plus haut possible compte tenu d'une rupture potentielle des argiles sous jacentes et de disposer la membrane au-dessus.

En effet, aussitôt le vide réalisé, le coefficient de sécurité va augmenter par accroissement de la résistance au cisaillement dans le remblai.

Les contraintes effectives $\sigma_{v}^{\prime}$ et $\sigma_{h}^{\prime}$ vont augmenter de la même valeur $\mathrm{nP}_{\mathrm{a}}$ et déplacer les cercles de Mohr vers la droite. On peut aussi considérer que tout se passe comme si la courbe intrinsèque du remblai était décalée vers la gauche de $\mathrm{nP}_{\mathrm{a}}$ c'est-à-dire que le remblai se comporte comme s'il avait une cohésion.

$$
\mathrm{C}_{\mathrm{m}}=\mathrm{nP}_{\mathrm{a}} \cdot \operatorname{tg} \phi
$$

dont la valeur est proche de $50 \mathrm{KPa}$. C'est ce qui se passe dans un paquet de café sous vide.

Cette cohésion apparente est toujours présente quelles que soient les déformations.

Cette valeur peut modifier énormément le coefficient de sécurité. Dans un cas réel cité dans les exemples de 


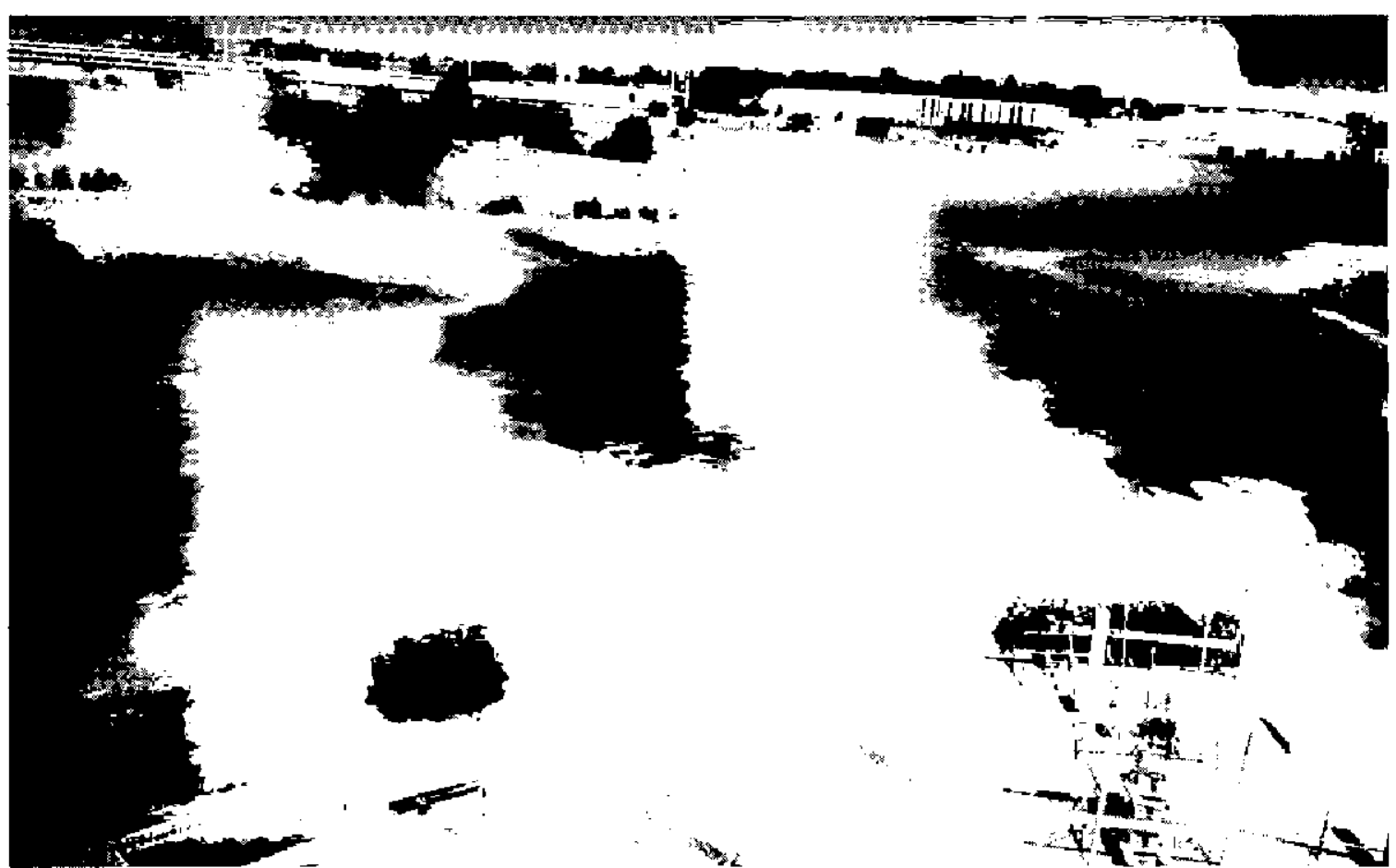

Fig. 15. - Membrane sous vide à proximitó d'un bätiment sur pieux.

Fig. 15. - Vacuum application in viscinity of a building on pites
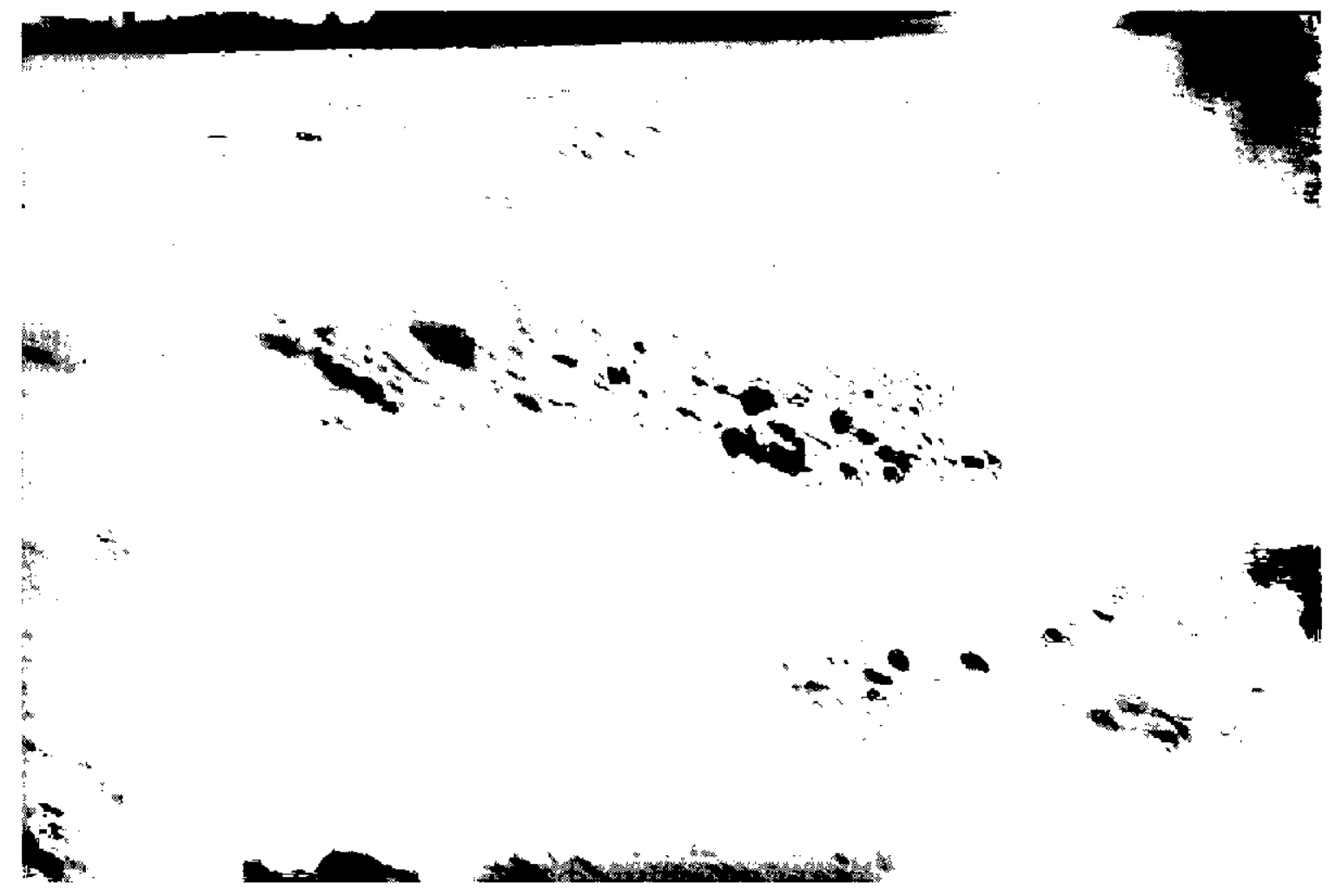

Fig. 16. - Un remblal sous vide.

Fig. 16. - Application of vacuum on coarse grained fill.

réalisation, le moment résistant dans les tourbes pour la hauteur de remblai maximale de $2,40 \mathrm{~m}$ était de $2760 \mathrm{KN} \cdot \mathrm{m} / \mathrm{mj}$ et la cohếsion provenant du vicle entre $A$ et $A^{\prime}$ ajoutait un moment résitant de 1540 $\mathrm{KN} . \mathrm{m} / \mathrm{ml}$ soit plus de $50 \%$.

\section{UTILISATIONS POSSIBLES DU PROCÉDÉ}

Indépendamment de la mise en place de la mem. brane posée sur le sol, il est possible de combiner 


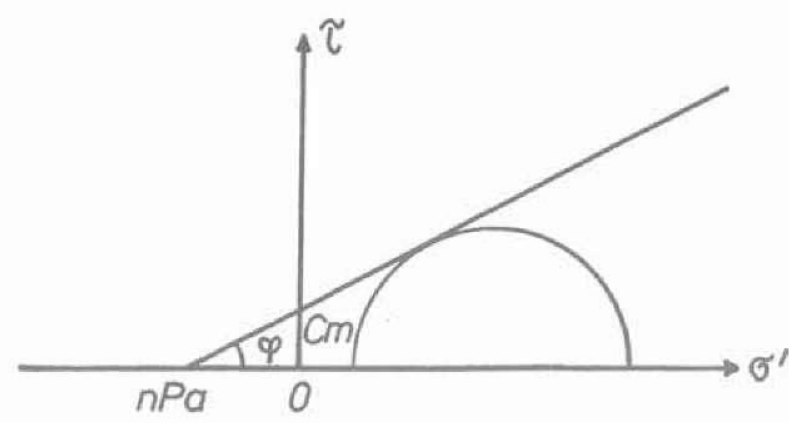

$$
\mathrm{Cm}=n P a \times \operatorname{tg} \varphi \simeq 50 \mathrm{kPa}
$$

Fig. 17. - Cohésion fictive dans les remblais due au vide. Fig. 17. - Apparent cohesion due to vacuum application.

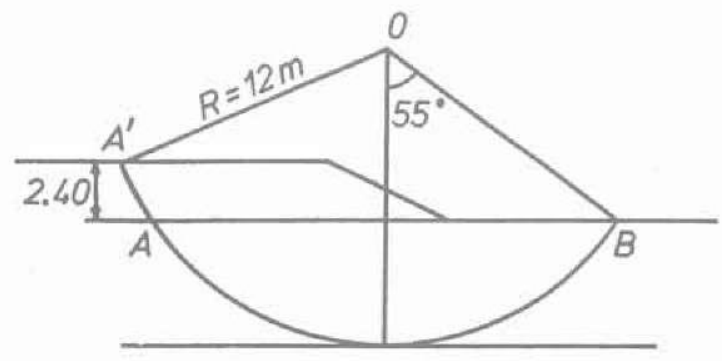

$$
\begin{aligned}
& \widehat{A B} \quad M r=2760 \mathrm{kN} \cdot \mathrm{m} \quad \text { pour } \mathrm{Cu}=10 \mathrm{kPa} \\
& \widehat{A A^{\prime}} \mathrm{Mr}=2.8 \times 12 \times 50=1540 \mathrm{kN} \cdot \mathrm{m}
\end{aligned}
$$

Fig. 18. - Amélioration du moment résistance due au vide dans le remblai.

Fig. 18. - Increment of resisting moment due to vacuum application in fill.

cette technique avec le préchargement de deux façons.

\subsection{Combinaison avec de l'eau}

Il suffit de réaliser des merlons périphériques et de remplir avec de l'eau en profitant de l'étanchéité de la membrane (fig. 19).

6.2. Combinaison avec un préchargement par remblais, c'est la méthode qui va être utilisée à Ambes : - mise en place d'un remblai à la cote maximale permise par le sol;

- pose de la membrane et pompage, ce qui va apporter une cohésion fictive par augmentation isotrope des contraintes effectives dans le remblai ;
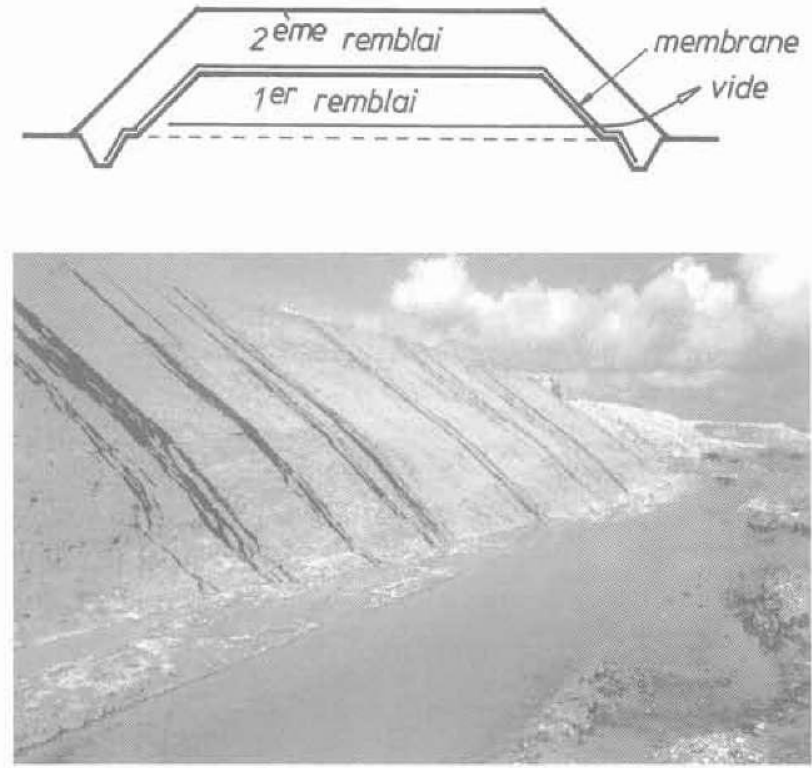

Fig. 20. - Remblai + membrane 1re phase.

Fig. 20. - Fill covered by a $1^{\text {st }}$ phase membrane.

Résumé des durées de pompage appliquées sur différents chantiers.

\begin{tabular}{|l|l|l|l|l|}
\hline $\begin{array}{c}\text { No } \\
\text { chantier }\end{array}$ & $\begin{array}{c}\text { Durée } \\
\text { de } \\
\text { pompage }\end{array}$ & $\begin{array}{c}\text { Maille } \\
\text { drains } \\
\text { rectangulaires }\end{array}$ & $\begin{array}{c}\mathrm{Cc} \\
\frac{i+e 0}{\text { moyen (1) }}\end{array}$ & $\begin{array}{c}\% \quad \text { (2) } \\
\text { Tassement }\end{array}$ \\
\hline 1 & 4 mois & 1,5 & 0,21 & 12,5 \\
2 & 4,5 mois & 1,8 & 0,17 & 8,5 \\
3 & $3 \quad$ mois & 1,5 & 0,22 & 9,5 \\
4 & 2,5 mois & 1,3 & 0,27 & 13,5 \\
5 & 3,5 mois & 1,5 & 0,18 & 7,5 \\
6 & 3,5 mois & 1,5 & 0,2 & 10,5 \\
7 & $3 \quad$ mois & 1,4 & 0,56 & 18 \\
8 (3) & 2,5 mois & 1,3 & 0,1 & 4 \\
\hline
\end{tabular}

N.B.

1. II s'agit d'une moyenne des essais réalisés lors de l'étude préalable, cette moyenne étant pondérée par l'épaisseur des couches auxquelles s'applique l'essal avec toutes les imprécisions d'une telle moyenne.

2. Il s'agit d'un tassement moyen mesuré par les boules de tassement, rapporté à la profondeur moyenné des drains dans la couche compressible.

3. Sol probablement en partie surconsolidé.

- mise en place d'une deuxième couche de remblai pour finir après tassement à la cote projet.

Tous les drains sont des drains circulaires en PVC annelés perforés avec filtres type Bidim.

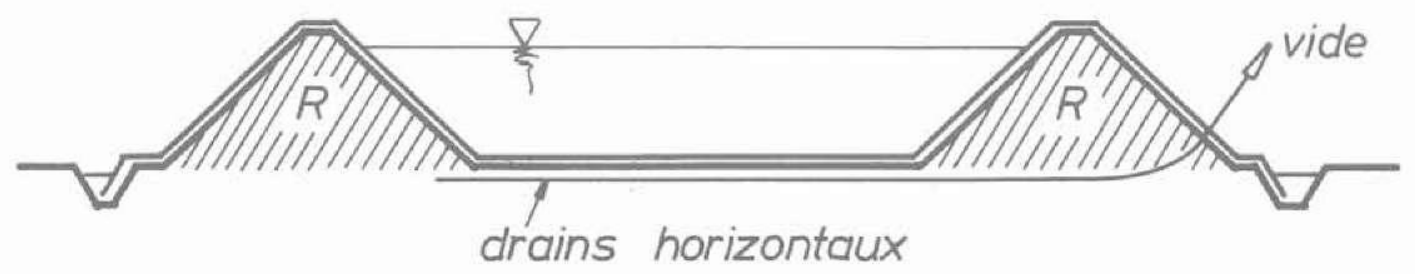

Fig. 19. - Vide + eau.

Fig. 19. - Vacuum + water 